\title{
Chondroblastic Osteosarcoma in A 13 Year Old Child
}

Sonia Bai J K ${ }^{1}$

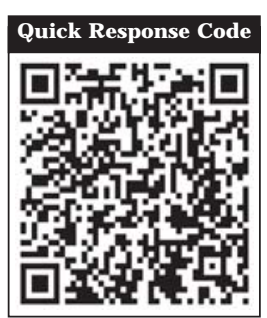

doi: $10.5866 / 2014.641713$

1Post Graduate Student Department of Oral Pathology,

G.Pullareddy Dental College And Hospital,

GPR Nagar, Nandyal Road, Kurnool, India

\section{Article Info:}

Received: J uly 8, 2014

Review Completed: August 9, 2014

Accepted: November 10, 2014

Available Online: J anuary, 2015 (www.nacd.in)

(C) NAD, 2015 - All rights reserved

\section{Email for correspondence:}

drsoniasinghrajput@gmail.com

\begin{abstract}
:
Primary neoplasms of human skel eton are rare, accounting for $0.2 \%$ of overall human tumor burden. Osteosarcoma is a malignant mesenchymal tumor, predominantly occurring in long bones and occasionally in the maxillofacial region. Osteosarcoma accounts for $15-35 \%$ of all primary bone tumors and represent 4-8\% of osteosarcomas of jaw. Here a case of chondroblastic variant of osteosarcoma in a 13 year old child on the left side of palate is reported.
\end{abstract}

Key words: Mesenchymal tumor, woven bone, osteosarcoma, long bones.

\section{INTRODUCTION:}

Osteosarcoma (OS) is an mesenchymal malignant bone tumor characterized by formation of disorganized immature woven bone or osteoid tissue with a reported incidence of $1: 100,000$ per year. ${ }^{1,2}$ Osteosarcomas account for $15-35 \%$ of all primary bone tumors and constitute $4-8 \%$ in the jaw. It is the most common non-hemopoietic primary malignant bonetumor of children and adolescence. ${ }^{3}$ Osteosarcomas of jaw are diagnosed two decades and later than sarcomas of long bone with a peak incidence between 20-40 years. Osteosarcomas of the head and neck originate from soft tissues and have lower metastatic rate than osteosarcomas of long bones. ${ }^{2}$ OS of jaw occurs mostly in males with a ratio of $1.5: 1$ and peak incidence is observed in $3-4^{\text {th }}$ decade. ${ }^{3}$ Common site of occurrence is body of mandible followed by alveolar ridge of maxilla and maxillary sinus. ${ }^{4}$ Typically the patient presents clinically as a swelling, pain, paresthesia and loosened teeth. ${ }^{5}$ Computed tomography helps in better diagnosis than conventional radiography. ${ }^{4}$ Osteosarcomas should be considered in differential diagnosis of expansile lesions of jaws. Early diagnosis and adequate surgical resection are the keys to high survival rates. ${ }^{4}$

\section{CASE REPORT:}

A 13-year-old male patient presented with a chief complaint of pain and swelling on the left side of the face with a corresponding swelling inside the oral cavity since one month. History revealed that swelling started one month ago on the left side of the palate spontaneously and gradually increased in size and associated with pain. There was no significant medical, dental or family history.

\section{Indian Journal of Dental AdVAnCEMEnTS} Journal homepage: www. nacd. in 
On inspection, a single diffuse extra-oral swelling is seen on the middle third of the face on left side (figure 1 ). The swelling was round to oval in shape measuring about $3 \times 4 \mathrm{~cm}$. The swelling extended superiorly up to $2 \mathrm{~cm}$ bel ow the lower eyelid and inferiorly $2 \mathrm{~cm}$ lateral to the corner of the mouth. It extended anteriorly up to the alae of nose, posteriorly $4 \mathrm{~cm}$ upto infront of the ear. The surface of swelling was smooth. There was no evidence of ulceration or sinus formation.

On intra-oral examination, swelling was found on the left side of the palate crossing the midline. Swelling was noted extending anteriorly from palatal rugae and posteriorly upto the maxillary tuberosity of size $5 \times 6 \mathrm{~cm}$ (figure 2) and laterally obliterating the buccal vestibule in relation to $25,26,27$ region. The surface over swelling was normal and swelling was tender on palpation. The conventional radiograph revealed a typical sunburst appearance (figure 3). There was widening of the periodontal space around the involved maxillary teeth, suggestive of tumor infiltration into PDL space. Provisional diagnosis was given as central giant cell granuloma.

Incisional biopsy was done and the tissue was sent for histopathological examination (figure 4). The Hand E stained decal cified section showed areas of woven bone with irregularly arranged deeply stained osteoblasts. Sheets of tumor osteoid with malignant chondroid tissue showing cellular pleomorphism (figure 4 and 5) and mitotic figures were evident. Areas showing primitive mesenchymal tissue were also evident in the sections. Based on histopathological findings, a diagnosis of chondroblastic osteosarcoma was given. After diagnosing the lesion as chondroblastic osteosarcoma, the case was referred to higher oncology center for further evaluation and treatment.

\section{DISCUSSION:}

Osteosarcoma(OS) is a true mal ignant neoplasm of bone, which may occur as a central, juxtacortical or peripheral lesion. ${ }^{6}$ OS is characterized by production of osteoid tissue by mesenchymal tumor cells. ${ }^{3}$ It is the second most common malignant bone tumor after multiple myeloma. ${ }^{3}$ OS accounts for $15-$ $35 \%$ of all primary bone tumors followed by chondrosarcoma and Ewing's sarcoma. ${ }^{1}$ OS of jaw bones are rare and account for $5-6 \%$ of all osteosarcomas with an estimated incidence of 0.07 cases per 100,000 per year. ${ }^{5}$
OS are generally classified into two types; primary and secondary. ${ }^{6}$ Etiology of primary OS is unknown and considered as may be due to genetic influence or environmental factors. Secondary OS occurs in older patients and predisposing factors include paget's disease, fibrous dysplasia of bone, trauma, chronic osteomyelities, bone infracts and late sequelae to crani ofacial irradiation. ${ }^{1,7}$ It also has been associated with metallic implants, joint prosthesis and in some genetic syndromes, Li Fraumeni syndrome, hereditary retinoblastoma and Rothmund- Thomson syndrome. ${ }^{1}$ Genetic mutations in tumor suppressor gene P53 and mutated retinoblastoma gene also found to be an etiological factor for the development of OS. This suggests an association between the occurrence of this neoplasm and increased cellular activity. ${ }^{8}$ OS occurs more commonly in males with peak incidence of 3-4 decade. Mandible has been reported to be a more likely location than the maxilla in many extensive reports. ${ }^{9}$ Primary OS represents a heterogenous group of malignant bone tumor characterized by diverse histological features, clinical, and biological behavior. ${ }^{10}$

OS of the jaws differ from long OS of long bones in several aspects: A) seen mostly in 3-4 decade (1015 years later than mean age of occurrence of OS of long bones). B) No bimodal distribution. C) More in Males ( I $n$ bones, equal predilection). D) Swelling is major complaint in OS of jaw where as pain in OS of long bones. E) Better prognosis. F) Less metastatic spread (6-51\%) in jaws and metastasis in long bones(78-90\%). ${ }^{11}$

Nearly $70 \%$ of OS display a multitude of cytogenetic abnormalities including haploidy in chromosomes 1p11-p13,1q11-q12,1q21-q22,11p14p15,14p11-p13,15p11-p13,17p, and 19q13; gain of chromosome 1; loss of chromosome 9,10,13 and 17; and amplification of chromosomes 6p12-p21,17p11 and 12q13-q14. ${ }^{12}$

Variants of OS recognized by WHO differ in location,clinical behavior and degree of cellular atypia in that majority are intramadullary (conventional) OS and remaining are surface OS which are further subdivided into paraosteal, periosteal and high grade surface OS.13.14

Osteosarcoma being a rare presentation in the oral cavity can easily be misdiagnosed. ${ }^{4}$ Clinically present with pain, swelling and tooth mobility. Radiographic findings vary from sclerotic to mixed 


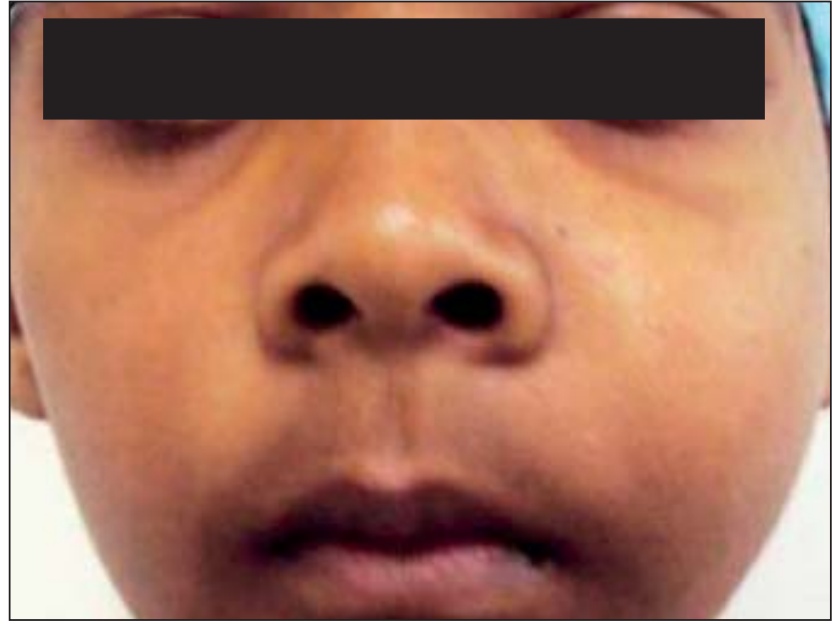

Figure 1: A single diffuse extra-oral swelling is seen on the middle third of the face on left side

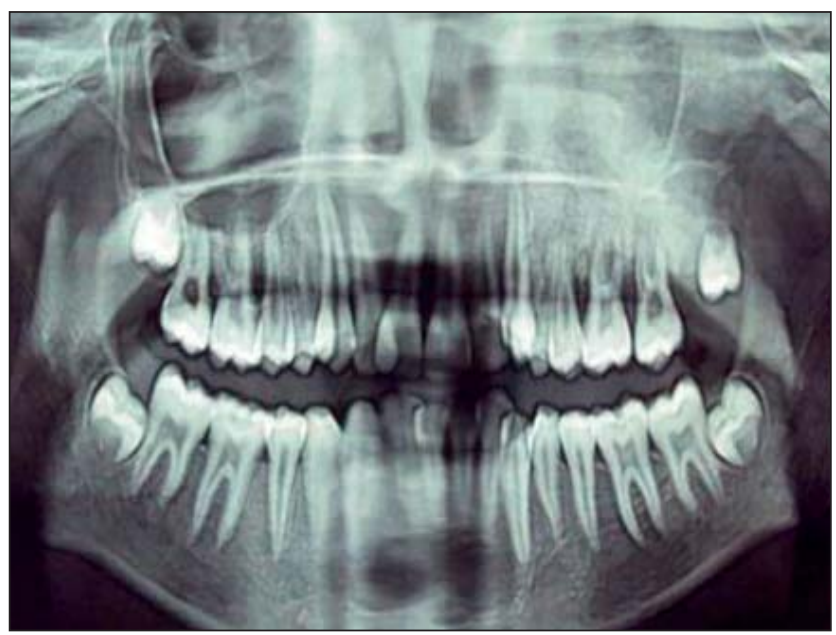

Figure 3: OPG Showing a typical sun-burst appearance

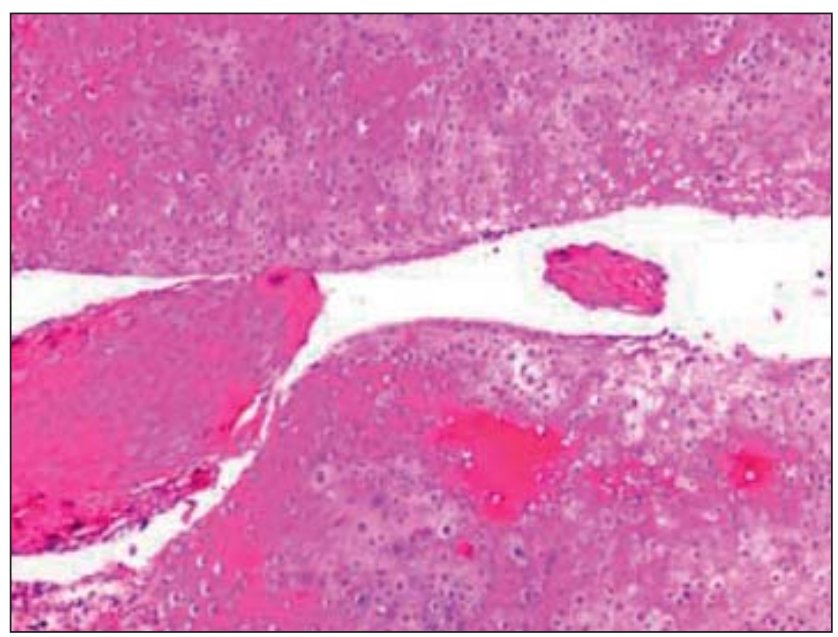

Figure 5: H\&E section showing areas of woven bone with irregularly arranged deeply stained osteoblasts

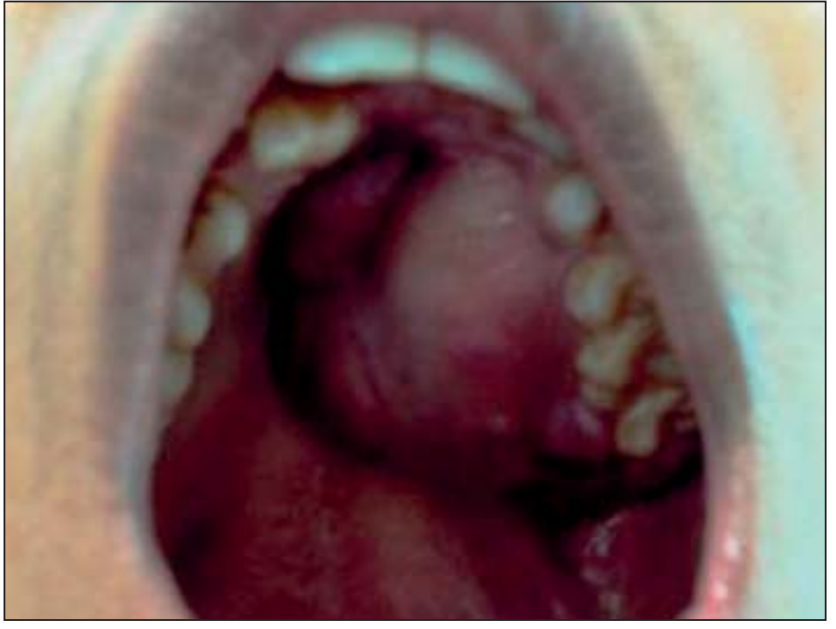

Figure 2: Swelling noted on the left side of palate extending from mid palatal raphae to the maxillary tuberosity.

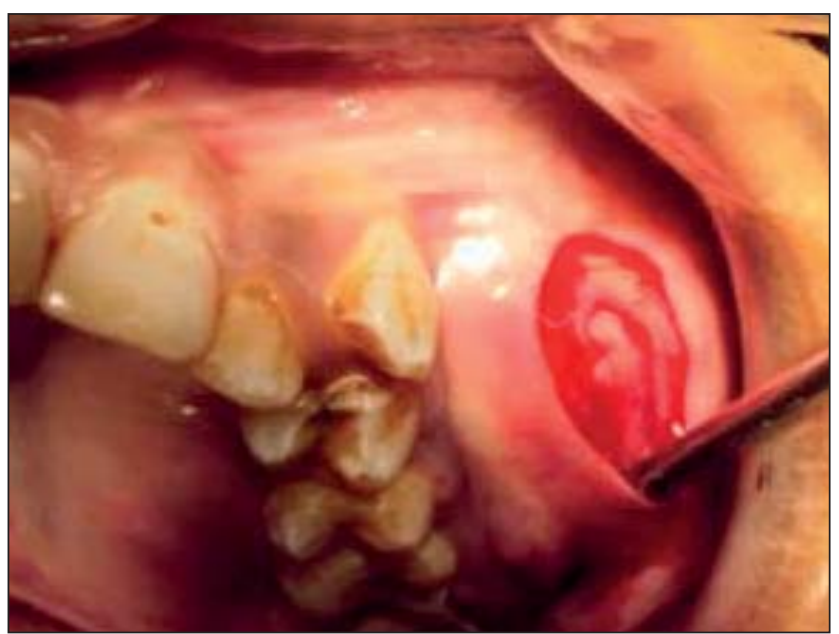

Figure 4: Surgical region where incisional biopsy was carried out.

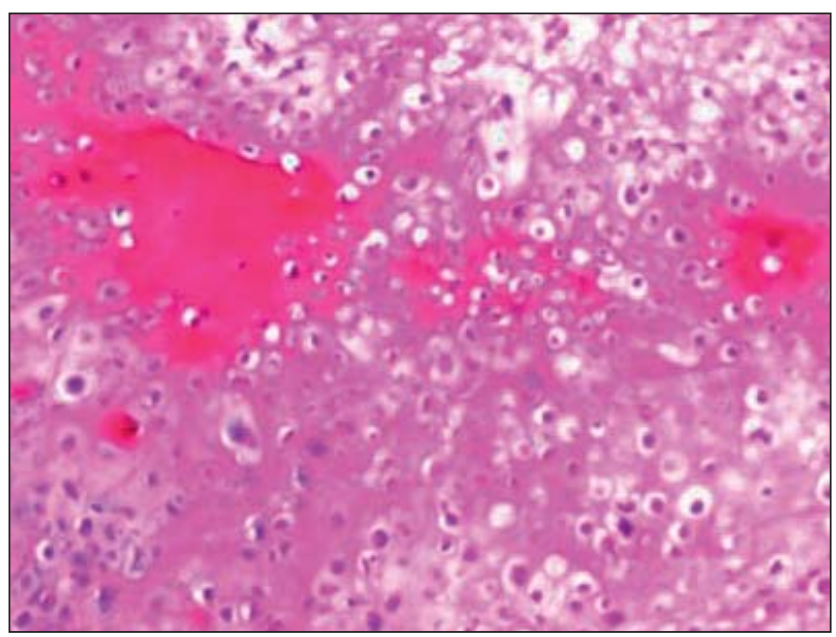

Figure 6: $\mathrm{H} \& \mathrm{E}$ section showing sheets of tumor osteoid with malignant chondroid tissue showing cellular pleomorphism (X10). 
sclerotic to radiolucent. "classic" sunray or sunburst appearance due to osteophytic bone production best demonstrated in occlusal radiograph and CT Scan. ${ }^{15}$ Panoramic radiograph may show Garrington's sign - widening of periodontal space around affected teeth with tapered resorption of tooth roots due to infiltration of tumor. ${ }^{16}$

Histologically OS of jaws are classified into osteoblastic, chondroblastic and fibroblastic depending upon the amount of the osteoid, cartilage or collagen fibres produced by the tumor. ${ }^{17}$

Staging of the tumor incorporates the degree of differentiation, distant metastasis and to estimate the prognosis of the patient. ${ }^{18}$ The system used most commonly for bone sarcomas is Enneking System which is based on grade (G) of thetumor, local extent of the primary tumor $(\mathrm{T})$, and metastasis $(\mathrm{M}){ }^{6}$

Immunhistochemistry (IHC) helps in differentiating chondrosarcoma from chondroblastic osteosarcoma as it is positivefor vimentin, EMA, S100 and rarely cytokeratin where as chondrosarcoma is positive for vimentin and S100 only. ${ }^{19}$

Most common site for metastasis is lung which accounts for $20 \%$. Risk of distant metastasis with OS of the head and neck varies, but death usually results from extension into the skull.

OS can be treated by radical or conservative surgery complemented by radiotherapy and/or chemotherapy. Overall prognosis is $25-50 \%$ with 5 year survival rate. ${ }^{17}$

\section{CONCLUSION:}

Osteosarcoma is an ancient disease in which many aspects still not understood. Excluding hematopoietic neoplasms, osteosarcoma is the most common type of malignancy which originates within bone and produce osteoid or immature bone. OS should always be considered in the differential diagnosis of expansile lesions of thejaws. Only early detection and proper treatment is the key to reduce morbidity and significantly improves prognosis. For proper management, emphasis should belaid on the aggressiveness of the lesion for early identification and diagnosis followed by prompt treatment.

Identifying the genes and signal transduction pathways through more molecular research may hel $p$ in the development of newer diagnostic markers and help in better prognosis and patient survival in the future.

\section{REFERENCES:}

1. Antony George, Varghese Mani, Sunil S, Sreenivasan BS, Devi Gopakumar. Osteosarcoma of maxilla: - a case of missed initial diagnosis. J OMFP 2010; 1(1): 33-38.

2. Tommaso Cutilli, Secondo Scarsella, Desiderio Di Fabio, Antonio Olivo, Pasqualino Cargini. High grade chondroblastic and fibroblastic osteosarcoma of the upper jaw. Annals of Maxillofacial Surgery; 2011; 1(2):176.

3. Antony George, Varghese Mani. Gnathic osteosarcomas: review of literature and report of two cases in maxilla. J OMFP 2011;15(2):138-143.

4. NM Praveena, G Maragathavalli. Osteosarcoma of maxilla. J Ind Acad Rad 2012; 24(3):236-238.

5. Christian Frei, Michael M.Bornstein, Edouard Stauffer, Tateyuki Lizuka, Daniel Buser. Osteosarcoma of the maxilla and maxillary sinus: a case report. Quintessence International 2004; 35(3):228-233.

6. Mayur Chaudhary, Shweta Dixit Chaudhary. Osteosarcoma of jaws. J OMFP 2012;16(2): 233-238.

7. Brackenridge CJ. A statistical study of sarcoma complicating Paget's disease of bone in three countries. Br J Cancer 1979; 40:194-200.

8. Sushrut B Vaidya, Srivalli Nadarajan, J yotsna S Galinde, Alok S Bhardwaj. J uxtacortical osteosarcoma of mandible. Annals Maxillofac Surgery 2011; 1(2):172-175.

9. ME Donaldson, JR Geist, TD Daley. Osteosarcoma of the jaws in children. Int J Paed Dent 2004; 14:54-60.

10. Soares RC, Soares AF, Souza LB, Santos AL, Pinto LP. Osteosarcoma of mandible initially resembling lesion of dental periapex: a case report. Braz J Otorhinolaryngol 2005; 71:242-245.

11. Price C, J effree GM. Metastatic spread of osteosarcoma. $\mathrm{Br} J$ Cancer 1973; 28:515.

12. Tang N, Song W, Luo J , Haydon RC, He TC. Osteosarcoma development and stem cell differentiation. Clin Orthop Relat Res 2008; 466:2114-2130.

13. Schajowicz F, Sissons HA, Sobin LH. The world health organization's Histologic classification of bone tumors: a commentary on the second edition. Cancer 1995; 75:12081214.

14. Schajowicz F. Historical typing of tumors of bone: world health Organization international classifi cation of tumors. Berlin: Springer Verlag 1993.

15. Zarbo RJ , Carlson ER. Malignancies of thejaws. In, Regezi J A, Sciubba J J , J ordan RK (ed). Oral Pathology, 5th edition. Saunders, 2008; 315-321.

16. Angela C. Bone Pathology. In, NevilleBW, Damm DD, Allen CM, Bouquot J E, (ed). Oral \& Maxill ofacial Pathology, 3rd edition. Saunders, 2009; 660-664.

17. Meet Kamal, Parwinder Kaur, Gurkiran Kaur, Shally Gupta. Osteosarcoma of mandible a rare entity- a case report. Ind J Dent Sci 2013; 2(5):68-70.

18. Raymond AK, Ayala AG, Knuutila S. Conventional osteosarcoma. In: Fletcher CD, Unni KK, Mertens F, editors. Pathology and Genetics of Tumors of Soft Tissue and Bone. $1^{\text {st }}$ ed. Lyon: IARC Press; 2002. pg 264-270.

19. Akpolat N, Yildirim H, Poyraz K. Sacral chondroblastic osteosarcoma misdiagnosed as chondrosarcoma and chordoma. Turk J Med Sci 2007; 37:243-249. 